University of Wollongong

Research Online

Faculty of Engineering and Information

Faculty of Engineering and Information

Sciences - Papers: Part B

Sciences

2019

\title{
Effects of foot orthoses on dynamic balance and basketball free-throw accuracy before and after physical fatigue
}

Wing-Kai Lam

Guangzhou Sport University, Shenyang Sport University, Li Ning Sports Science Research Centre, gilbertlam@li-ning.com.cn

Winson Lee

University of Wollongong, ccwlee@uow.edu.au

Siu-On Ng

Li Ning Sports Science Research Center, University of Hong Kong

Yi Zheng

Capital University of Physical Education and Sports

Follow this and additional works at: https://ro.uow.edu.au/eispapers1

Part of the Engineering Commons, and the Science and Technology Studies Commons

Research Online is the open access institutional repository for the University of Wollongong. For further information contact the UOW Library: research-pubs@uow.edu.au 


\title{
Effects of foot orthoses on dynamic balance and basketball free-throw accuracy before and after physical fatigue
}

\author{
Abstract \\ While it is not uncommon for athletes to use foot orthoses to relieve pain and improve sports \\ performance, little has been known about their effects on basketball performance. Free-throw basketball \\ shooting is very important. However, fatigue deteriorates postural balance which might decrease free- \\ throw shooting performance. This study investigated the effects of foot orthoses on dynamic balance \\ and accuracy performance during free-throw shooting before and after physical fatigue was induced. \\ Thirteen male recreational basketball players were tested with two foot orthoses (medial-arch support \\ versus flat control) and fatigue conditions (before and after fatigue), when they performed standard free- \\ throw shooting on a force platform. Results revealed that fatigue significantly increased coefficient of \\ variance of medial-lateral center of pressure (CoP) excursion when participants worn flat control orthoses \\ $(p<0.05)$. Meanwhile, foot orthoses improved dynamic balance during shooting as they significantly \\ reduced total resultant and anterior-posterior sway excursions as well as resultant and anterior-posterior \\ CoP velocities, and base of support area. Although this study found that fatigue and orthoses did not \\ significantly affect the scores gained by free-throw shooting, the significant improvements in dynamic \\ balance during shooting with the use of foot orthoses could have considerable impact on motor control \\ during basketball shooting.

\section{Disciplines} \\ Engineering | Science and Technology Studies

\section{Publication Details} \\ Lam, W., Lee, W. Chiu Chun ., Ng, S. \& Zheng, Y. (2019). Effects of foot orthoses on dynamic balance and \\ basketball free-throw accuracy before and after physical fatigue. Journal of Biomechanics, 19 \\ 109338-1-109338.
}


1 Running Title: Dynamic balance in free-throw

3 Title: Effects of foot orthoses on dynamic balance and basketball free-throw

4 accuracy before and after physical fatigue

5

6 Lam, W., Lee, W. Chiu Chun ., Ng, S. \& Zheng, Y. (2019). Effects of foot 7 orthoses on dynamic balance and basketball free-throw accuracy before 8 and after physical fatigue. Journal of Biomechanics, 19 109338-1-

9109338.

10 


\section{Abstract}

12 While it is not uncommon for athletes to use foot orthoses to relieve pain and

13 improve sports performance, little has been known about their effects on

14 basketball performance. Free-throw basketball shooting is very important.

15 However, fatigue deteriorates postural balance which might decrease free-throw

16 shooting performance. This study investigated the effects of foot orthoses on

17 dynamic balance and accuracy performance during free-throw shooting before

18 and after physical fatigue induced. Thirteen male recreational basketball players

19 were tested with two foot orthoses (medial-arch support versus flat control) and

20 fatigue conditions (before and after fatigue), when they performed standard free-

21 throw shooting on a force platform. Results revealed that fatigue significantly

22 increased coefficient of variance of medial-lateral center of pressure $(\mathrm{CoP})$ excursion

23 when participants worn flat control orthoses $(p<0.05)$. Meanwhile, foot orthoses

24 improved dynamic balance during shooting as they significantly reduced total

25 resultant and anterior-posterior sway excursions as well as resultant and anterior-

26 posterior CoP velocities, and base of support area. Although this study found that

27 fatigue and orthoses did not significantly affect the scores gained by free-throw

28 shooting, the significant improvements in dynamic balance during shooting with

29 the use of foot orthoses could have considerable impact on motor control during

30 basketball shooting.

32 Word Count: 197 (Abstract), 3432 (Main text)

33 Keywords: Insole, orthotic, postural stability, sports, shooting 


\section{1. Introduction}

36 An estimated 450 million people participate in basketball worldwide (FIBA

37 2018). In a basketball game, 16-21\% of the playing time is involved in high

38 intensity activities (Ben Abdelkrim et al., 2010), with execution of various jump,

39 cutting, and lateral movements (Ben Abdelkrim et al., 2007; Lopezosa-Reca et

40 al., 2017). Such intensity of activities can easily produce physical fatigues

41 causing reduced strength and coordination, which is associated with inferior

42 performances in jump, agility, passing and shooting accuracy (Lyons et al., 2006;

43 Padulo et al., 2015).

44 Free-throw shooting is extremely important, which directly influences the

45 team's success. Physical fatigue poses greatest challenge in shooting accuracy

46 (Padulo et al., 2015; Okazaki et al., 2015). It has been documented that physical

47 fatigue could deteriorate dynamic balance (Zemkova 2014), which in turn led to

48 lower rifle (Ball et al., 2003; Mononen et al., 2007) and archery (Mason and

49 Pelgrim, 1986) shooting accuracy. Similarly, basketball free-throw shooting also

50 requires players to maintain a stable position with very little sway while

51 simultaneously shooting the ball. A plausible strategy that can enhance dynamic

52 balance may potentially improve basketball free-throw shooting.

53 It is not uncommon for athletes to use foot orthoses to relieve pain

54 (Hirschumuller et al., 2011) and improved sport performance (Banwell et al.,

55 2014; Mills et al., 2010). More than 50\% of basketball players were reported to

56 use foot orthoses in competition and training (Losito, 2010), as it can improve

57 balance, foot alignment, strain and load on soft tissues, and receptor sensory on

58 the foot plantar in various populations (Davidson, 2010; Ho et al., 2019; Lusardi

59 and Nielsen, 2007). Biomechanically, foot orthoses with medial arch-support 
60 increase the foot-insole contact area and pressure at the medial longitudinal arch

61 of a foot, enhancing somatosensory inputs over the plantar foot. Previous studies

62 showed that they allow wearers to walk comfortably while enabling them to

63 maintain postural stability using a narrow base of support, which was considered

64 to be more challenging than a wider base of support (Hrysomallis, 2011;

65 Zemkova 2014).

66 Static and dynamic balance is the ability to maintain postural stability and

67 orientation with center of mass over the base of support and body at rest and in

68 motor task, respectively (Hrysomallis, 2011; Zemkova, 2014). Such postural

69 skills and stability can be developed through practice (Paillard 2014; Zemkova,

70 2014). Previous studies have found that poor shooters had inferior postural

71 stability than the good shooters (Zemkova, 2014) and that poor shooter

72 demonstrated higher center of mass velocity $(76.5 \mathrm{~m} / \mathrm{s})$ compared to good $(49.0$

$73 \mathrm{~m} / \mathrm{s}$ ) and elite $(44.9 \mathrm{~m} / \mathrm{s}$ ) shooters (Verhoeven and Newell, 2016). However, the

74 relationship among shooting performance, dynamic stability and use of foot

75 orthoses in basketball free-throw is not yet established.

76 This study examined the effects of foot orthoses on the dynamic balance

77 during free-throw shooting and accuracy of shooting before and after physical

78 fatigue. We expected that fatigue would induce inferior postural stability and

79 shooting performance. We also expected that arch-support foot orthoses would

80 improve dynamic balance and shooting performance when participants were in

81 fatigue conditions. The information can be insightful for coaches and sport

82 scientists who consider to use foot orthoses in training and competitions. 


\section{2. Methods}

\section{$85 \quad 2.1$ Participants}

86 A priori power analysis was performed to determine the appropriate

87 sample size of 8 for this study, based on an alpha of 0.05 and $80 \%$ power with

88 medium effect size (Cohen's $d=0.5$, Cohen 1988) calculated with pilot data to

89 detect postural stability between fatigue levels. Thirteen male basketball players

90 [mean age $21.4 \pm 3.3$ years; height $1.8 \pm 0.06 \mathrm{~m}$; mass $72.0 \pm 7.1 \mathrm{~kg}$ ] were recruited

91 for this study. Average competition experience was $4.5 \pm 3.7$ years. Only

92 participants having normal foot arches and foot lengths of US size 9 were

93 included. We used the pressure plate (Footscan 7, RSscan International,

94 Belgium) and Brannock foot measurement device (Brannock Device, Syracuse,

95 NY, USA) to confirm if the participants were normal foot arches. All participants

96 were right-handed basketball shooting and free of any inner ear infection, other

97 foot deformities, and lower extremity injuries for at least 6 months prior to the

98 start of the study. Ethical approval was granted by the Institutional Review Board

99 and written informed consent was obtained before data collection.

100

1012.2 Orthosis and shoe conditions

102 To eliminate foot size and footwear as confounding factors, all of the

103 participants were tested with the identical high-top basketball shoes (Wade 6, Li

104 Ning, Beijing, China) with US size 9.0 were used with the two orthoses

105 conditions (medial-arch support versus flat control) for this study. The tested

106 orthoses were fabricated with the insole milling machines (CNC Milling 
107 machine, Vulcan series, Sensor Medica, Italy) and the height of medial-arch

108 support and forefoot/rearfoot thickness were based upon previous studies (Alavi-

109 Mehr et al., 2018; Zhai et al., in press). The two orthoses were identical in terms

110 of material, thickness and hardness, except for the inclusion of medial-arch

111 support (see Fig. 1). The same orthotist quantified the hardness of the orthoses

112 across forefoot, midfoot and rearfoot regions using a Type $\mathrm{C}$ shore durometer

113 (1600 Asker SP-698 Rex Durometers, Rex Gauge Co., IL, USA) (Lam et al.,

114 2017; Nin et al., 2015). Five measurements taken at each of the five dots from

115 each respective regions were averaged and confirmed both orthoses have the

116 same hardness across different regions.

\subsection{Equipment and laboratory setting}

121 All fatigue protocols and free-throw shooting were performed in our

122 biomechanical laboratory. To replicate indoor basketball court, force plate and its

123 surrounding surfaces were covered with standard basketball court surface and the

124 free-throw distance and height of basketball rim were set according to the

125 standard of International Basketball Federation (FIBA, 2018). Posturography

126 data were collected by synchronised force platform (AMTI, Watertown, USA,

127 sampling frequency of $1000 \mathrm{~Hz}$ ) and motion analysis system of ten cameras

128 (Oxford Metrics Ltd, Oxford, sampling frequency of $200 \mathrm{~Hz}$ ) during basketball

129 free-throw shooting. Six reflective markers were placed over the posterior (heel),

130 anterior (toe), and lateral (fifth metatarsal) aspects of each of left and right shoes

131 to define the area of base of support during free-throw trials (DiDomenico et al., 
132 2015). To prevent hindering the movements in test and fatigue-inducing protocol,

133 no reflective markers were attached at any other body parts. Another high-speed

134 camera (Casio EX-F1, Casio, Japan) at a sampling rate of $300 \mathrm{~Hz}$ was placed

135 perpendicular to the shooting arm of the participant to determine the start and

136 end points of each shooting trial. Both force plate and high speed camera were

137 synchronised when determining the first basketball contact during a basketball

138 dribbling on the two measuring systems.

$140 \quad 2.4$ Fatigue-inducing protocol

141 To induce physical fatigue, all participants were asked to perform both

142 Yo-Yo Intermitted Recovery protocol - level 1 (YYIR1, Bangsbo et al., 2008)

143 and consecutive maximal vertical jumps (Zemkova and Hamar, 2010) in this

144 study. The YYIR1 consisted of multiple 2 x 20 m shuttle runs with a short $10 \mathrm{~s}$

145 recovery period between sets, as previously described (Bangsbo et al., 2008). In

146 brief, participants were instructed to complete the full 20 run and turn indicated

147 by the audio beep sound, and return to the starting point. There was an 10s

148 recovery period between every shuttle run interval. The starting speed was 10.0

$149 \mathrm{~km} / \mathrm{hr}$ and increased to $12 \mathrm{~km} / \mathrm{hr}, 13 \mathrm{~km} / \mathrm{hr}$, then increasing by $0.5 \mathrm{~km} / \mathrm{hr}$

150 thereafter. The participants continued running as long as they were unable to

151 continue. The maximum running speed sustained by participants was $14.8 \pm 1.0$

$152 \mathrm{~km} / \mathrm{h}$

153 Consecutive maximal vertical jumps involved at least 10 times maximal-

154 effort vertical jumps, which were shown to sufficiently deteriorate the dynamic

155 balance (Zemkova and Hamar, 2010). After 10 consecutive maximal jumps, the

156 participants were required to continue the maximum-effort jumps until their heart 
157 rate attained $85 \%$ of the maximum heart rate, which was found to be experienced

158 by most players in basketball competitions (Mclnnes et al., 1995). The maximum

159 heart rate was determined when multiply individual age by 0.7 , then subtract the

160 results from 207 (Gelish equation, Pescatello et al., 2013). Heart rate was

161 recorded with a heart rate monitor system (Polar PCX3, Polar, Kempele,

162 Finland).

\subsection{Procedures}

165 The experimental procedure is shown in Fig. 2. After having the

166 anthropometrical measurements, participants wore a new pair of standard socks

167 and test basketball shoes (Wade 6, Li Ning, Beijing, China) to prevent the

168 potential influences due to the socks and footwear (Ho et al., 2019; Lam et al.,

169 2018; Liu et al., 2017; Nin et al., 2016). They were then instructed to administer

170 a 10-min self-instructed warm-up including stretching and jogging and then

171 familiarise themselves with the testing tasks and footwear condition, which was

172 comparable with previous studies on basketball footwear and orthoses (Ho et al.,

173 2019; Lam et al., 2018; Liu et al., 2017; Nin et al., 2016).

178 using a standard ball (size 7), from the free-throw line (4.0m from the front part

179 of the basket) into a standard basketball rim (height $=3.05 \mathrm{~m}$; circumference $=$

$180 \quad 0.45 \mathrm{~m}$ ) in each of the two orthosis conditions (medial-arch support and flat). The

181 participants were then asked to perform the fatigue-inducing protocol, which 
182 included YYIR1 and consecutive maximum vertical jumps, separated by a 30-

183 second standing rest on the shooting area. The participants then performed 20

184 free-throw shooting trials. The trial was discarded if the obvious slippage and

185 discontinuity of movement was present. After the first orthosis condition, the

186 maximal jump protocol was repeated before the second orthosis measurements

187 were made to maintain the physical fatigue state (Fig. 2). The orders of the use of

188 the foot orthoses were randomised in all conditions across participants.

$190 \quad 2.6$ Data reduction

191 The analysed period of a free-throw was defined manually from the

192 lowest point of elbow joint to the point of basketball release of the shooting arm.

193 A spline interpolation was performed for minor missing data using three frames

194 of data before and after the missing data. All signals were smoothed with a fourth

195 order Butterworth bidirectional filter (DiDomenico et al., 2015). The cut-off

196 frequencies of were determined using a residual analysis (Winter, 1990). The

197 marker trajectories were identified using Vicon Clinical Manager Software

198 (Oxford Metrics Ltd, Oxford) and then transferred into Visual3D software (C-

199 Motion Inc.) for definition of area of base of support during free-throw shooting

200 trial (DiDomenico et al., 2015).

201 The following posturography variables were selected to denote the

202 dynamic balance during each shooting trial (Zemkova, 2014): maximum range of

203 resultant, medial-lateral (ML) and anterior-posterior (AP) center of pressure

204 (CoP) excursion (sway range; mm), total resultant, ML and AP CoP excursion

205 (sway excursion; mm), mean resultant, ML and AP sway velocity along the CoP

206 path (sway velocity; $\mathrm{mm} / \mathrm{s}$ ), and 95\% ellipse sway area included within the COP 
207 path (sway area; $\mathrm{mm}^{2}$ ). Resultant CoP travelling distance was defined as the

208 distance of the present CoP coordinate relative to the coordinate of the previous

209 frame. The sway range was defined as the largest coordinates subtracted the

210 smallest coordinates in each shooting cycle. Total excursion was defined as sum

211 of the resultant CoP path covered the whole shooting period. The $95 \%$ ellipse

212 sway area was defined as an estimated ellipse to the CoP data that encompassed

$21395 \%$ of the data. The mean sway velocity was defined as rate of change of CoP

214 path of a body between frames. Maximum range of resultant, ML and AP CoP

215 excursions, ML and AP sway velocities and 95\% ellipse sway area are

216 commonly used to assess postural balance of human (Distefano et al., 2009). The

217 literature reported very little about the postural balance of basketball players.

218 However, these parameters have been studied in other sports which included

219 soccer (Paillard and Noe, 2006), dancers (Gerbino et al., 2007), swimmers and

220 gymnasts (Hrysomallis, 2011). Small CoP excursion, variability of AP and ML

221 excursion and velocity, and sway ellipse area were indicative of good balance.

222 The shooting performance was quantified using a six-point scale to

223 examine the differences in performance over sessions (Lam et al., 2009a). In

224 brief, 5 was awarded for a 'clean' basket (i.e., "swish”); 4 for rim and in; 3 for

225 backboard and in; 2 for rim and out; 1 for backboard and out; and 0 for a

226 complete miss. The shooting score of each shooting trial was recorded by the

227 same researcher and then averaged for each fatigue and orthosis condition as the 228 dependent variable for shooting performance. All participants were reminded of

229 the scoring system and encouraged to score as many points as possible (Lam et 230 al., 2009a). 


\section{Data analysis}

233 To assess the main effects and interaction of fatigue and orthosis conditions

234 (independent variables) on dynamic balance and shooting performance

235 (dependent variables), a $2 \times 2$ two-way (Orthosis x Fatigue-level) ANOVA with

236 repeated measures was performed on both mean and coefficient of variation

237 (CV) in each of the dependent variables. The CV was defined as the standard

238 deviation divided by mean, which allowed comparing the degree of variability

239 across variables (Reed et al., 2002). The CVs across 20 free-throw shooting trials

240 were calculated for respective fatigue and orthosis conditions for each of the

241 participants for subsequent analyses. Bonferroni corrected post-hoc tests were

242 performed accordingly, if the two-way ANOVA indicated a significant

243 interaction among the independent variables. Level of significance was set at

244 0.05. All statistical analyses were performed using the SPSS statistical analysis

245 computer program package, version 21.0 (SPSS, Inc., Chicago, IL, U.S.A.).

\section{3. Results}

$248 \quad 3.1$ Dynamic balance during free-throw shooting

249 The Orthosis x Fatigue-level (2 x 2) ANOVA with repeated measures revealed

250 significant main effects of Orthosis $(P<0.05$, Table 1$)$, but no main effect of

251 Fatigue-level or interaction between Orthosis and Fatigue-level $(P>0.05)$ in

252 mean values of CoP data. The medial arch-support orthoses produced

253 significantly smaller total resultant and AP sway excursions, resultant and AP

254 CoP velocities, and base of support area than the flat control orthoses $(P<0.05$,

255 Table 1). 
260 interactions on maximum sway range in ML CoP excursion $(P<0.05)$. The

261 simple main effect revealed that participants wearing flat control orthoses

262 displayed higher CV ML CoP excursion in post-fatigue (41\%) compared to pre-

263 fatigue shooting condition $[31 \%, P<0.05,95 \% \mathrm{CI}=-9.85(-20.14$ to 0.45$)]$,

264 while there was no significant difference when wearing medial arch-support

265 orthoses $[P>0.05,95 \% \mathrm{CI}=5.65(-4.49$ to 15.78$)]$.

\subsection{Basketball free-throw scores}

270 The Orthosis $x$ Fatigue-level $(2 \times 2)$ ANOVA with repeated measures did

271 not reveal any significant effect of Orthosis, Fatigue-level nor interaction

272 between Orthosis and Fatigue-level for mean and CV of shooting performance

$273(P>0.05$, Table 3).

\section{Discussion}

This study examined whether foot orthoses would influence the dynamic

279 balance and free-throw accuracy before and after physical fatigue. Our results

280 indicated that physical fatigue significantly increased coefficient of variance 
281 (CV) of ML CoP excursion in the condition of flat control orthoses. This is

282 consistent with previous studies, which suggested that fatigue had detrimental

283 effect on balance control in a rifle shooting position (Bermejo et al., 2017),

284 marksmanship shooting score (Gil-Cosano et al., 2018), throwing speed and

285 accuracy (Nuno et al., 2016). Once medial-arch support orthoses were used,

286 physical fatigue no longer had any significant effects on all analysed dynamic

287 balance parameters. This indicated the positive effect of orthoses on dynamic

288 balance during basketball free-throw shooting.

289 There was further evidence supporting the benefits of medial-arch

290 support orthoses on dynamic balance during basketball free-throw shooting. The

291 orthoses induced smaller sway excursions, sway velocities, and base of support

292 area. Smaller CoP motion and sway were indicative of better postural balance

293 (Hrysomallis, 2011). In addition, the medial arch-support orthoses allowed

294 participants to maintain stability using a narrow area of support, which was

295 considered to be more difficult than a wider area of support (Zemkova 2014;

296 Zemkova and Hamar, 2010). While previous studies have indicated the positive

297 effects of foot orthoses on dynamic balance of people with old age (Ma et al.,

298 2018) and neuro-musculoskeletal disorders (Lusardi and Nielsen, 2007), this

299 study further demonstrated the beneficial effects in basketball playing.

300 Interestingly, while the orthoses support the medial midfoot influencing foot

301 motions primarily in the frontal plane (Davidson, 2010; Lusardi and Nielsen,

302 2007), our findings showed medial arch-support orthoses was associated with

303 dynamic balance in anterior-posterior direction, but not medial-lateral direction.

304 This can be explained by the shooting movement which is executed primarily in 
305 sagittal plane. In the future, more investigation should be carried out before a

306 viable conclusion can be made.

307 It is believed that physical fatigues are related to poor performances such

308 as reduced jump, agility, passing, throwing and shooting accuracy (Gil-Cosano et

309 al., 2018; Lyons et al., 2006; Padulo et al., 2015; Nuno et al., 2016). In the

310 present study, the free-throw accuracy was not different across fatigue levels,

311 which contrasted with the previous fatigue studies investigating on basketball

312 shooting (e.g., upper limb fatigue studies: Erculj and Supej, 2009; Padulo et al.,

313 2015). One possible reason could be related to the use of different shooting

314 distances among studies. One previous study (Erculj and Supej, 2009) had

315 participants performing three-point shot (7.2m) from a basket and showed

316 significant changes in shoulder and upper arm biomechanics with growing

317 fatigue. Our study had participants performing standard free-throw shot (4.0m)

318 from the basket and showed no differences in dynamic balance and shooting

319 performance between two fatigue levels. Physical fatigue may pose greater

320 challenge to longer-distance shooting rather than shorter distances. Studying the

321 effects of upper limb and lower limb fatigues on shooting performance in various

322 shooting distances would provide insightful knowledge in developing training

323 regimes.

324 Our findings which indicated that medial arch-support orthoses improved

325 dynamic balance but not shooting accuracy were in line with previous studies

326 done on the jump shot (Chen et al., 2016) and free-throw performance

327 (Zemkova, 2014). These findings also showed that more pronounced side-to-side

328 CoM movement (i.e., less postural stability) does not affect the accuracy of

329 basketball free-throw shots (Zemkova, 2014). Such findings were contrast with 
330 shooting performances of other sports, which included rifle and archery, in which

331 dynamic balance was found to be significantly related to shooting accuracy

332 (Hrysomallis, 2011). Such discrepancies could be due to the movement

333 complexity between rifle archery shooting and basketball free-throw shooting.

334 While rifle and archery involves smaller number of joint coordination (e.g.,

335 fingers), basketball involves larger number of joint coordination (e.g., knee,

336 shoulder, elbow, wrist) which makes accuracy less influence by dynamic

337 balance. Principal components and minimal number of movement components

338 techniques would help to quantify the degree of self-organisation in basketball

339 shooting (Kelso, 1995; Lam et al., 2009b).

340 Although our studies did not measure the comfort perception variables,

341 comfort perception has been received considerable interest by coaches and sports

342 scientists. Foot orthoses would increase the perceived comfort and reduce the

343 pain/discomfort (Esfandiari et al., 2018; Lucas-Cuevas et al., 2014; Mills et al.,

344 2010). In rugby, the increase of comfort perception was related to higher actual

345 performance (Kinchington et al., 2012) and lower incidence of injuries

346 (Kinchington et al., 2011) in both competition and training. Additionally, higher

347 comfort perception was associated with improved running economy (Luo et al.,

348 2009) and impact attenuation (Mills et al., 2010) during running. The evidence

349 from these previous studies suggested that foot orthoses improved sport

350 performance via increased comfort perception on the wearers. Since comfort

351 perception is highly related to past experience, age, gender, and task difficulty

352 (e.g., Kong \& Bagdon, 2010; Lam et al., 2011; Schubert et al., 2011),

353 methodology for valid assessments of footwear comfort require further

354 investigation (Lam et al., 2011, 2013, 2015). More research is also required to 
355 determine the efficacy of the foot orthoses on shooting and other functional

356 movements in basketball.

357 When interpreting our results, it is important to consider some limitations in

358 this study. First, single male group was recruited in this study, which did not

359 allow generalization to other gender or playing positions. Different playing

360 positions in basketball players would have distinct physical attributes (Koklu et

361 al., 2011), which might affect the motor control strategies and performance

362 response to fatigues and foot orthoses. Second, this study did not measure any

363 psychological factors/attention demand during sports performance. Future studies

364 may investigate if they influence fatigue level, dynamic balance and sports

365 performance.

366

367 5. Conclusion

368 Medial arch-support orthoses improved dynamic balance during shooting

369 as they significantly reduced CoP excursion, velocities of movement and base of

370 support area. Although this study found that fatigue and foot orthosis did not

371 significantly affect the scores gained by free-throw shooting, the significant

372 improvements in postural balance during shooting with the use of medial arch-

373 support orthoses could be insightful for motor control in basketball shooting. 
375

376

377

378

379

380

381

382

383

384

385

386

387

388

389

390

391

392

393

\section{References}

Alavi-Mehr, S.M., Jafarnezhadgero, A., Salari-Esker, F., Zago, M., 2018. Acute effect of foot orthoses on frequency domain of ground reaction forces in male children with flexible flatfeet during walking. The Foot 37, 77-84.

Ball, K.A., Best, R.J., Wrigley, T.V., 2003. Body sway, aim point fluctuation and performance in rifle shooters: inter- and intra-individual analysis. J. Sports Sci. 21, 559-566.

Bangsbo, J., Iaia, F.M., Krustrup, P., 2008. The Yo-Yo intermittent recovery test: a useful tool for evaluation of physical performance in intermittent sports. Sports Med. 38, 37-51.

Banwell H., Mackintosh S., Thewlis D., 2014 Foot orthoses for adults with flexible pes planus: a systematic review. J. Foot Ankle Res. 7, 23.

Ben Abdelkrim, N., El Fazaa, S., El Ati, J., 2007. Time-motion analysis and physiological data of elite under-19-year-old basketball players during competition. Br. J. Sports Med. 14, 69-75.

Ben Abdelkrim, N, Castagna, C., El Fazaa, S., El Ati, J., 2010. The effect of players' standard and tactical strategy on game demands in men's basketball. J. Strength Cond. Res. 24, 2330-2342.

Bermejo, J.L., Garcia-Masso, X., Paillard, T., Noe, F., 2017. Fatigue does not conjointly alter postural and cognitive performance when standing in a shooting position under dual-task conditions. J Sports Sci. 36, 429-435.

Chen, Y.J., Chen, Z.R., Cheng, H.Y., Song, C.Y., Chen, Y.H., Peng, H.T., 2016. The influence of arch support insole on basketball jump shot. Paper presented at the 34th International Conference on Biomechanics in Sports, Tsukuba, Japan.

Cohen, J., 1988. Statistical power for the behavioural sciences. $2^{\text {nd }}$ ed. Hillsdale (NJ): Lawrence Erlbaum Associates.

Davidson, D.M., 2010. Prefabricated insoles and modifications in sports medicine. In M.B. Werd, E.L Knight (eds), Athletic footwear and orthoses in sports medicine. Springer, New York, pp.89-94.

Didomenico, A., McGorry, R.W., Banks, J.J., 2015. Factors affecting time-tocontact calculations during quiet standing. Motor Control 19, 1-9. 
407

408

409

410

Distefano, L.J., Clark, M.A., Padua, D.A., 2009. Evidence supporting balance training in healthy individuals: A systematic review. J. Strength Cond. Res. 23, 2718-2731.

Erculj, F., Supej, M., 2009. Impact of fatigue on the position of the release arm and shoulder girdle over a longer shooting for an elite basketball player. J. Strength Cond. Res. 23, 1029-1036.

Esfandiari, E., Sanjari, M.A, Jamshidi, A.A., Kamyab, M, Yazdi, H.R. 2018. Knee osteoarthritis at the early stage: The four-week effect of lateral wedge insole on pain and risk of falls. Med. J. Islam Repub. Iran. 32, 17.

FIBA., 2018. Facts \& Figures [online]. Federation Internationale de Basketball Amateur. Retrieved January 28, 2018, from http://www.fiba.basketball/presentation\#|tab=element_2_1

Gerbino, P.G., Griffin, E.D., Zurakowski, D., 2007. Comparison of standing balance between female collegiate dancers and soccer players. Gait Posture 26, 501-507.

Gil-Cosano, J.J., Orantes-Gonalez, E., Heredia-Jimenez, J., 2018. Effect of carrying different military equipment during a fatigue test on shooting performance. Eur. J. Sport Sci. 19, 186-191.

Ho, M., Kong, P.W., Chong, L., Lam, W.K., 2019. Foot orthoses alter lower limb biomechanics but not jump performance in basketball players with or without flat feet. J. Foot Ankle Res. 12, 24.

Hirschumuller, A., Baur, H., Muller, S., Helwig, P., Dickhuth, H.H., Mayer, F., 2011. Clinical effectiveness of customised sport shoe orthoses for overuse injuries in runners: a randomised controlled study. Br. J. Sports Med. 45, 959-965.

Hrysomallis, C., 2011. Balance ability and athletic performance. Sports Med. 41, 221-232.

Kelso, J.A.S., 1995. Dynamic patterns: The self-organization of brain and behaviour. Cambridge, MA: MIT Press.

Kinchington, M., Ball, K., Naughton, G., 2011. Effects of footwear on comfort and injury in professional rugby league. J. Sports Sci. 29, 1407-1415.

Kinchington, M., Ball, K., Naughton, G., 2012. Relation between lower limb comfort and performance in elite footballers. Phys. Ther. in Sport 13, 27-34. 
440 Koklu, Y., Alemdaroqlu, U., Kocak, F.U., Erol, A.E., Findikoqlu, G., 2011.

441 Comparison of chosen physical fitness characteristics of Turkish professional

442 basketball players by division and playing position. J. Hum. Kinet. 30, 99-

$443 \quad 106$.

444 Kong, P.W., Bagdon, M., 2010. Shoe preference based on subjective comfort for

445 walking and running. J. Am. Podiatr. Med. Assoc. 100, 456-462.

446 Lam, W.K., Fung, H.T., Poolton, J.M., 2015. Footwear fit perception with or

447 without attention. Footwear Sci. 7, 181-190.

448 Lam, W.K., Lee, W.C.C., Lee, W.M., Ma, C.Z.H., Kong, P.W., 2018. Segmented

449 forefoot plate in basketball footwear: Does it influence performance and foot

450 joint kinematics and kinetics? J. Appl. Biomech. 34, 31-38.

451 Lam, W.K., Maxwell, J.P., Masters, R.S.W., 2009a. Analogy versus explicit

452 learning of a modified basketball shooting task: Performance and kinematic

453 outcomes. J. Sports Sci. 27, 171-191.

454 Lam, W.K., Maxwell, J.P., Masters, R.S.W., 2009b. Analogy learning and the

455 performance of motor skills under pressure. J. Sport Exer. Psy. 31, 337-357.

456 Lam, W.K., Ng, W.X., Kong, P.W., 2017. Influence of shoe midsole hardness on

457 plantar pressure distribution in four basketball-related movements.

$458 \quad$ Res.Sports Med. 25, 37-47.

459 Lam, W.K., Sterzing, T., Cheung, J.T., 2011. Reliability of a basketball specific

460 testing protocol for footwear fit and comfort perception. Footwear Sci. 3,

$461 \quad 151-158$.

462 Lam, W.K., Sterzing, T., Cheung, J.T., 2013. Influence of protocol complexity

463 on fit perception of basketball footwear. Footwear Sci., 5, 155-163.

464 Liu, H., Wu, Z., Lam, W.K., 2017. Collar height and heel counter-stiffness for

465 ankle stability and athletic performance in basketball. Res. Sports Res. 25,

$466 \quad 209-218$.

467 Lopezosa-Reca, E., Gijon-Noqueron, G., Morales-Asencio, J.M., Cervera-Marin,

468 J.A., Luque-Suarez A., 2017. Is there any association between foot posture

469 and lower limb-related injuries in professional male basketball players? A

$470 \quad$ cross-sectional study. Clin. J. Sport Med. doi:

$471 \quad$ 10.1097/JSM.0000000000000563 
472 Losito, J.M., 2010. Basketball and Volleyball. In M.B. Werd, E.L. Knight (eds).

473 Athletic footwear and orthoses in sports medicine, Springer, NY, USA. pp.

474 275-282.

475 Lusardi, M.M., Nielsen, C.C., 2007. Orthotics and prosthetics in rehabilitation.

476 Elsevier, PA, USA.

477 Lucas-Cuevas A.G., Pérez-Soriano, P. Priego-Quesada J.I., Llana-Belloch S

478 (2014) Influence of foot orthosis customisation on perceived comfort during

479 running, Ergon. 57(10), 1590-1596.

480 Luo, G., Stergiou, P., Worobets, J., Nigg, B., 2009. Improved footwear comfort

481 reduces oxygen consumption during running. Footwear Sci. 1, 25-29.

482 Lyons, M., Al-Nakeeb, Y., Nevill, A., 2006. The impact of moderate and high

483 intensity total body fatigue on passing accuracy in expert and novice

484 basketball players. J. Sports Sci. Med. 5, 215-227.

485 Ma, C.Z.H., Wong, D.W.C., Wan, A.H.P., Lee, W.C.C., 2008. Effects of

486 orthopedic insoles on static balance of older adults wearing thick socks Prost.

487 Orthot. Int. 42, 357-362.

488 Mason, B.R., Pelgrim, P.P., 1986. Body stability and performance in archery.

$489 \quad$ Exel 3, 17-20

490 McInnes, S.E., Carlson, J.S., Jones, C.J., McKenna, M.J., 1995. The

491 physiological load imposed on basketball players during competition. J.

492 Sports Sci. 13, 387-397.

493 Mills, K., Blanch, P., Chapman, A.R., McPoil, T.G., Vicenzino, B., 2010. Foot

494 orthoses and gait: a systematic review and meta-analysis of literature

495 pertaining to potential mechanisms. Br. J. Sports Med. 44, 1035-1046.

496 Mononen, K., Konttinen, N., Viitasalo, J., 2007. Relationship between postural

497 balance, rifle stability and shooting accuracy among novice rifle shooters.

498 Scand. J. Med. Sports 17,180-185.

499 Nin, D.Z., Lam, W.K., Kong, P.W., 2015. Effect of body mass and midsole

500 hardness on kinetic and perceptual variables during basketball landing

501 manoeuvres. J. Sports Sci. 34, 1-10.

502 Nuno, A., Chirosa, I.J., can der Tillaar, R., Guisado, R., Martin, I., Martinez, I.,

503 Chirosa, L.J., 2016. Effects of fatigue on throwing performance in

504 experienced team handball players. J. Hum. Kinet. 54, 103-113. 
505

506

507

508

509

510

Okazaki, V.H. A., Rodacki, A.L.F., Satern, M.N., (2015). A review on the basketball jump shot. Sports Biomech. 14, 190-205.

Padulo, J., Attene, G., Migliaccio, G.M., Cuzzolin, F., Vando, S., Ardigò, L.P., 2015. Metabolic optimisation of the basketball free throw. J. Sports Sci. 33, 1454-1458.

Paillard, T., 2014. Sport-specific balance develops specific postural skills. Sport Med. 44, 1021-1023.

Paillard, T.H., Noe, F., 2006. Effect of expertise and visual contribution on postural control in soccer. Scand. J. Med. Sci. Sports 16, 345-348.

Pescatello, L.S., Riebe, D., Thompson, P.D., 2013. ACSM's guidelines for exercise testing and prescription. Lippincott Williams \& Wilkins, Philadelphia.

Prieto, T.E., Myklebust, J.B., Hoffmann, R.G., Lovett, E.G., Myklebust, B.M. 1996. Measures of postural steadiness: differences between healthy young and elderly adults. IEEE Trans.Biomed. Eng.43, 956-966.

Reed, G.F., Lynn, F., Meade, B.D., 2002. Use of coefficient of variation in assessing variability of quantitative assays. Clin. Diagn. Lab. Immunol. 9, 1235-1239.

Schubert, C., Oriwol, D., Sterzing, T., 2011. Gender and age related requirements of running shoes: a questionnaire on 4501 runners. Footwear Sci. 3(Suppl 1), s148-s150.

Van Emmerik, R.E.A., Miller, R.H., Hamill, J., 2014. Dynamical systems analysis of coordination. In D.G.E. Robertson, G.E. Caldwell, J. Hamill, G. Kamen, S.N. Whittlesey (eds), Research methods in biomechanics. Human Kinetics, Champaign,IL, pp. 291-317.

Verhoeven, F.M., Newell, K.M., 2016. Coordination and control of posture and ball release in basketball free-throw shooting. Hum. Mov. Sci. 49, 216-224. Winter, D.A., 1990. Biomechanics and motor control of human movements. New York: Wiley.

Wulf, G., Mercer, M., McNevin, N., Guadagnoli, M.A., 2004. Reciprocal influences of attentional focus on postural and suprapostural task performance. J. Motor Behavior 36, 189-199. 
537 Wulf, G., Shea, C., Park, J.H., 2001. Attention and motor performance:

538 preferences for and advantage of an external focus. Res. Quarterly Exer.

539 Sport. 72, 335-334.

540 Zhai, J., Wang J., Qiu Y., (in press). Whether flexible flatfoot needs the

541 treatment? An observation of plantar pressure effects on adults with flexible

542 flatfoot by wearing over the counter insoles when walking on level surface,

543 upstairs and downstairs. JAPMA.

544 Zemkova, E., Hamar, D., 2010 The effect of 6-week combined agility-balance

545 training on neuromuscular performance in basketball players. J. Sports Med.

546 Phys. Fitness 50, 262-267.

547 Zemkova, E., 2014. Sport-specific balance. Sports Med. 44, 579-590. 


\section{$550 \quad$ Figure Captions}

551 Fig. 1. A) Standard basketball shoe, B) Regions for hardness measurement, and

552 C) Dimensions of medial arch-support and flat orthoses.

554 Fig. 2. Overall experimental procedure.

556 Fig. 3. Interaction of coefficient of variation (CV) of M-L CoP excursion by

557 orthosis and fatigue-level conditions. MAS denotes medial arch-support orthoses. 
559

560

561

562 